\title{
A New Approach for GNSS-IR Snow Depth Monitoring With Slope Correction and Error Prediction
}

Peng Chen ( $\square$ chenpeng0123@gmail.com )

College of Geomatics, Xi'an University of Science and Technology, Xi'an 710054, China

Zheng Li

Xi'an University of Science and Technology

Naiquan Zheng

Xi'an University of Science and Technology

\section{Research Article}

Keywords: GNSS (Global Navigation Satellite System) technology, SONTEL network, RMSE (Root Mean Square Error)

Posted Date: January 22nd, 2021

DOI: https://doi.org/10.21203/rs.3.rs-150402/v1

License: (c) (i) This work is licensed under a Creative Commons Attribution 4.0 International License. Read Full License 


\title{
1 A new approach for GNSS-IR snow depth monitoring with 2 slope correction and error prediction
} \\ Peng Chen ${ }^{1, *}$, Zheng $\mathrm{Li}^{1}$, and Naiquan Zheng ${ }^{1}$ \\ 4 'College of Geomatics, Xi' an University of Science and Technology, Xi' an 710054, China \\ 5 "Peng Chen@ chenpeng0123@gmail.com
}

\begin{abstract}
With the continuous development of GNSS (Global Navigation Satellite System) technology, GNSS-IR (GNSS Interferometric Reflectometry) has become a research hotspot in the field of snow surface monitoring, and the accuracy and reliability have been initially verified. We focus on the reasons for the low accuracy of the existing GNSS-IR snow depth inversion. Therefore, we use the P351 station data in the PBO (Plate Boundary Observatory) network in the United States to monitor the snow depth during the four years from 2011 to 2015. The actual measured snow depth at station 490 in the SONTEL network is used as the true value for accuracy verification. We studied the relationship between the inversion error caused by the slope and the slope angle and the satellite elevation angle, and proposed a slope correction method. The results show that the RMSE (Root Mean Square Error) of snow depth inversion after slope correction is reduced from $12.1 \mathrm{~cm}$ to $10.7 \mathrm{~cm}$, the accuracy is improved by $11.6 \%$. In addition, it is found that there is an apparent correlation between the retrieved snow depth and the inversion error. With the increase of snow depth, the error gradually changes from positive to negative, and the absolute value of error still increases with the increase of snow depth after the error changes to negative. To this end, we introduce BPNN (Back Propagation Neural Network) to train the inversion snow depth and inversion error of the three snowfall periods from 2011 to 2014, then predicts and corrects the snow depth inversion error during the snowfall period from 2014 to 2015. The results show that the RMSE of the corrected GNSS-IR snow depth inversion is reduced from $10.7 \mathrm{~cm}$ to $5.7 \mathrm{~cm}$, and the accuracy is increased by $46.7 \%$. The overall accuracy of the GNSS-IR snow depth inversion is improved by $52.9 \%$ after the slope correction and the BPNN error prediction is performed, which further verifies the accuracy and effective of the approach that proposed by us.
\end{abstract}

Introduction

As an indispensable freshwater resource in the ecological environment of the earth, the distribution area and changes in reserves of snow are of considerable significance to the development of the ecosystem and society. On the one hand, the snow-melt water acts as a cold source for the atmosphere and can delay the process of seasonal transition. On the other hand, when the thickness of the snow reaches a certain level, it will cause traffic congestion and destroy the ecological environment. The monitoring of snow cover rate, snow depth or snow water equivalent can not only improve the ability of snow monitoring in snow prone areas, but also provide reliable information guarantee for social and economic development and environmental protection.

In recent years, with the continuous development and improvement of GNSS technology, GNSS-IR technology has gradually become a new means of surface snow monitoring. The rapid increase of GNSS tracking stations also provide a large number of reliable data sources for the research and application of GNSS-IR technology. Many scholars have carried out preliminary research on GNSS-IR, and the feasibility 
of snow depth inversion has been preliminarily verified. Martin-Neria ${ }^{1}$ first proposed the concept of PARIS (Passive Reflectometry and Interferometry System) that uses GPS (Global Positioning System) direct and reflected signals to perform interferometry. Comp and Axelrad ${ }^{4}$ improved a technique that mitigates specular multipath in GPS differential carrier phase measurements. It adaptively estimates the spectral parameters (frequency, amplitude, phase offset) of multipath in the associated SNR, and then constructs a profile of the multipath error in the carrier phase. Bilich and Larson ${ }^{2}$ proposed a method to remove the direct signal from the SNR data, and use the remaining SNR data to reflect the multipath environment. In the second year, they present a technique for mitigating specular multipath in GPS carrier phase measurements using the SNR, in which the frequency and amplitude content of non-stationary oscillations in SNR are modeled to extract multipath parameters ${ }^{3}$. Larson et al. ${ }^{7}$ used the SNR data of the GPS receiver to calculate the snow depth. The results show that the retrieved snow depth is consistent with the measured snow depth. Since then, they have also carried out relevant experiments on Retrieving Sea surface height, water content of vegetation and soil moisture with GPS receivers, and obtained excellent results ${ }^{8,9,18,19}$. Ozeki and $\mathrm{Heki}^{14}$ demonstrated that the geometry-free linear combination (L4), commonly used to study the ionosphere, can also be used to analyze multipath signatures, and investigated the possibility of inferring physical conditions of the snow surface using amplitudes of multipath signatures. Larson et al. ${ }^{10}$ analyzed the data of three GPS stations located in the Greenland ice sheet, and the results showed that the use of continuous GPS measurements on the ice sheet to monitor the vertical position of the antenna and the height of the reflecting surface can be a model of snow and snow density provide useful constraints Qian and Jin ${ }^{15}$ used SNR and phase of GLONASS to invert the snow depth, the snow depth results from GLONASS are almost similar with GPS. Combined GPS and GLONASS observations have no significant improvement on the precision, but improve the spatial resolution because of more satellites. Li et al. ${ }^{11}$ proposed a new method to estimate snow depth by using the single frequency GNSS signal combined by pseudo-range and carrier phase observations and a weighted method for combining multiple snow depth values generated by observations of multiple individual GNSS satellites. Yu et al. ${ }^{22}$ proposed two methods for inverting snow depth using dual GNSS receiving systems, one using dual-frequency carrier phase observation, and the other using a single-frequency carrier phase and pseudorange observation. Both methods can eliminate the influence of geometric distance and ionospheric delay. The experimental results show that the dual-receiver system can better use the GNSS signal carrier phase observations to estimate the snow depth, thereby improving data utilization. Wang et al. ${ }^{21}$ retrieve snow depth using Multi-GNSS data. A Multi-GNSS combination method based on robust regression is used to combine the inter-constellation inter-signal retrievals, and the Multi-GNSS combined retrievals show an improvement in precision, availability, and temporal sampling.

At present, with the continuous deepening of GNSS-IR technology research, GNSS-IR snow depth inversion technology has made a lot of progress. However, there are still some problems to be solved, and the accuracy of inversion needs to be further improved. The slope is a non-negligible factor in the GNSSIR snow depth inversion. Based on the study of the relationship between the error and the slope and elevation angle, we propose a slope correction method to reduce the inversion error caused by the slope. In addition, we study the correlation between the inversion error and the inversed snow depth, and proposes a method for modeling and forecasting the inversion error using BPNN (BP Neural Network). The P351 station of the PBO network is used to verify the accuracy and effectiveness of the two methods. 
This section introduces the data used in snow depth inversion and GPS tracking stations, gives the principle and main process of GNSS-IR snow depth inversion, and briefly introduces the BP neural network. Data

We use GPS L1 observation data from P351 station in PBO (Plate Boundary Observatory) network in the United States to conduct snow depth inversion from DOY (Day of year) 201, 2011 to DOY 200, 2015. The snow cover around the station is in the state of snow cover from the beginning of October to the end of May of the next year. The actual snow depth of station 490 of the SNOTEL network is used as the true value to verify the inversion results. P351 station belongs to the PBO network of the United States. It is located at $43.87441^{\circ} \mathrm{N}, 114.71916^{\circ} \mathrm{W}$, and altitude is $2,692 \mathrm{~m}$. The antenna height is $1.902 \mathrm{~m}$. The measured snow depth data comes from station 490 of the SNOTEL network, which is located at $43.87497^{\circ} \mathrm{N}$, $114.71363^{\circ} \mathrm{W}$, with an altitude of $2,676 \mathrm{~m}$ and a distance of $1.8 \mathrm{~km}$ from P351. Figure 1 shows the surrounding environment of the P351 station.

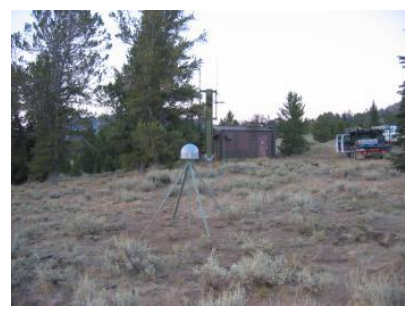

(a)

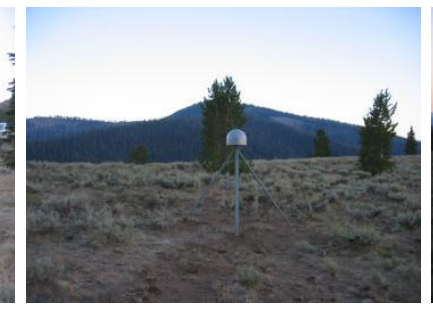

(b)

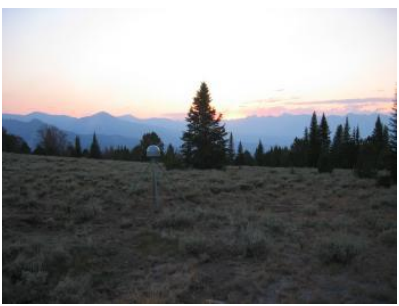

(b)

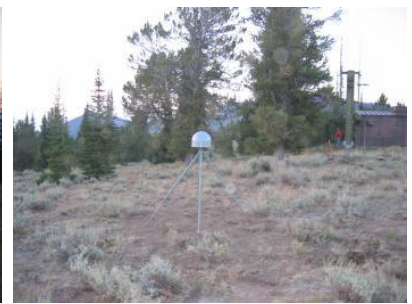

(d)

Figure 1. Environment map of P351 station. (a), (b), (c) and (d) shows the topography of east, west, north and south view of the station, respectively. (https://www.unavco.org/instrumentation/networks/status/ nota/photos/P351)

\section{GNSS-IR snow depth inversion principle}

GNSS receiver can receive two kinds of signals, one is the signal directly into the receiver, the other is the reflection signal caused by multipath effect. We can get the overall trend term of signal SNR data by using the second-order polynomial fitting, and remove it to get the SNR data of the reflected signal. Then the SNR data of the reflected signal is analyzed, and the distance from the antenna phase center to the reflector is calculated. Figure 2 is a schematic diagram of GNSS-IR snow depth inversion. $H$ is the receiver antenna height, that is, the vertical distance between the antenna phase center and the soil surface. $h$ is the vertical distance between the antenna phase center and the snow surface. sh is the snow depth. E is the incident angle of the direct signal into the receiver, that is, the satellite elevation angle ${ }^{12}$.

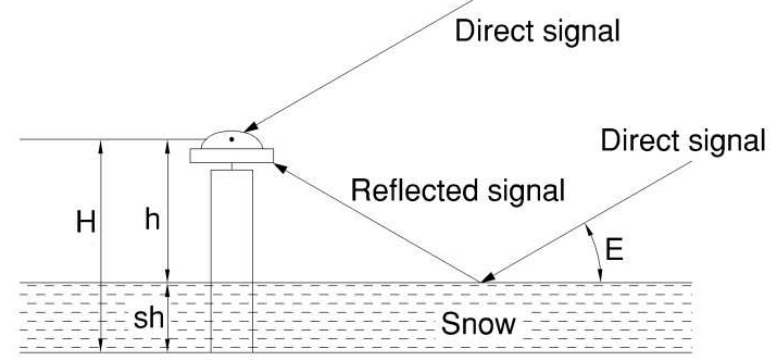

Figure 2. Schematic diagram of GNSS-IR snow depth inversion. 
The SNR data in the observation file of the geodetic GNSS receiver is the SNR data after mixing the direct signal and the reflected signal. The mathematical expressions of the SNR of the composite signal, the direct signal and the reflected signal are as follows ${ }^{13}$ :

$4 \quad S N R=A_{c}=A_{d}+A_{r}+2 \sqrt{A_{d} A_{r}} \cos \phi$

where $A_{c}$ is the SNR of the composite signal; $A_{d}$ is the SNR of the direct signal; $A_{r}$ is the SNR of the reflected signal; $A_{d} \gg A_{r} ; \phi$ is the phase difference between the direct signal and reflected signal. Since $A_{d}>A_{r}$, the direct signal SNR determines the overall trend of the composite signal SNR, and the direct signal SNR can be eliminated by fitting the overall trend of the composite signal SNR data ${ }^{13}$.

Figure 3 shows the SNR sequence of a continuous observation arc of PRN15 satellite on DOY 225, 2014 at P351 station. In the top panel, the blue curve is the composite signal SNR, the red curve is the overall trend of the fitting mixed SNR data. The red curve can be approximately regarded as the SNR of direct signal and removed. The brown curve is the satellite elevation angle. The bottom panel is the SNR residual data after removing the overall trend, which can be regarded as the reflected signal SNR. Then the snow depth can be retrieved by using the data. It can be seen from the figure that the SNR of a satellite is closely related to the elevation angle of the satellite, and it increases as the elevation angle increases. GNSS receiving antenna is mainly RHCP (right hand circular polarized) polarization. When the signal enters the receiver after reflection, it is mainly LHCP (left hand circular polarized), which can be well suppressed by antenna gain. When the incident angle of the reflected signal is less than 30 degrees, the main polarization is still RHCP, and the antenna cannot suppress the multipath effect well ${ }^{12}$. Therefore, in snow depth inversion, the elevation angle is set at $5^{\circ}-30^{\circ}$.
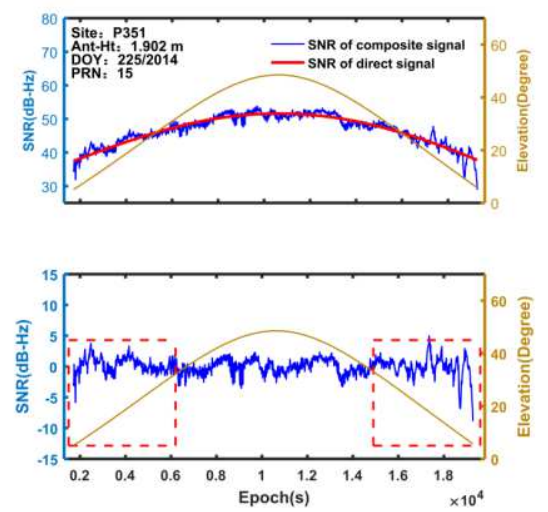

Figure 3. A sequence of signal SNR in a continuous observation arc of PRN15 satellite on DOY 225, 232014.

The mathematical expression between the reflected signal SNR and the height of the antenna phase center is as follows ${ }^{3,17}$ : 
1 distance between the antenna phase center and the reflecting surface, and $\varphi$ is the phase $\operatorname{shift}^{20}$. Let: $t=\sin E$

$2, f=\frac{2 h}{\lambda}$,then the (2) can be expressed as:

$3 \quad A_{r}=A \cos (2 \pi f t+\varphi)$

$4 \quad$ According to formula (3), we can get $f$ value by spectrum analysis or least square fitting of SNR 5 residual sequence, and finally calculate snow depth.

6 BP Neural Network

7 BPNN has a high degree of nonlinear mapping ability. As the most commonly used training algorithm, 8 BPNN is a gradient descent procedure that computes the values of the derivatives in an efficient way, and 9 modifies the weights according to a parameter known as the learning rate ${ }^{5}$. BPNN is composed of three layers, which are input layer, hidden layer and output layer. According to the number of hidden layers, it can be divided into a single hidden layer neural network and multiple hidden layer neural network. The single hidden layer training speed is fast, but the precision is low. When the training samples are small, the single hidden layer network can be selected for training. When the training samples are large and the influence factors are complex, the multi hidden layer BPNN is generally selected to ensure the accuracy.

The principle of the BPNN is that all the training samples are transmitted to the hidden layer through the input layer. The hidden layer learns and outputs the results according to the designed structure. When the error between the result and the expected value in the training sample meets the requirements, it is considered that the BPNN has learned the problem. Otherwise, it will learn again until the error reaches the requirements. Figure 4 shows the topological structure of the BPNN.

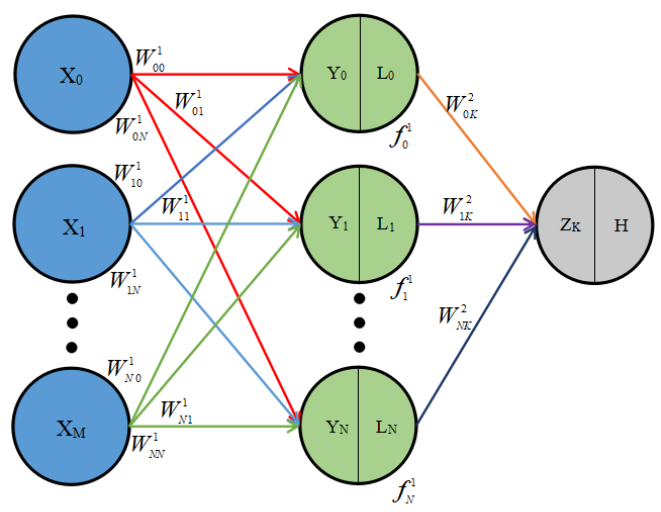

21 Figure 4. Schematic diagram of BPNN topology.

The mathematical principle of the forward propagation of BPNN is as follows ${ }^{6}$ :

$23 \quad Y_{j}=\sum_{i=0}^{M} W_{i j}^{1}+f^{1} M$

where $X_{i}$ is the input vector, $M$ is the number of input layer nodes and $i \in(0, M), \quad W_{i j}^{1}$ is the weighted 25 value between the $i$ th neurons in the input layer and the $j$ th neurons in the hidden layer, $f^{1}$ is the threshold parameter of the hidden layer, $Y_{j}$ is the node input value of the hidden layer and $j \in(0, N), \quad N$ and is the 
number of hidden layer nodes. The input value of each hidden layer node is converted to the output value $L_{j}$ of the corresponding hidden layer node through the nonlinear transfer function [16].

\section{Results and analysis}

To evaluate the accuracy of the GNSS-IR snow depth inversion results, we use use the Root Mean Square Error (RMSE) and the correlation coefficient (R) to evaluate the accuracy. The mathematical expression is as follows:

$$
\left\{\begin{array}{l}
\mathrm{RMSE}=\sqrt{\frac{\sum_{i=1}^{n}\left(X_{\mathrm{S}, i}-X_{\mathrm{G}, i}\right)^{2}}{n}} \\
\mathrm{R}=\frac{\sum_{i}^{n}\left(X_{\mathrm{G}, i}-\bar{X}_{\mathrm{S}, i}\right)}{\sum_{i}^{n}\left(X_{\mathrm{S}, i}-\bar{X}_{\mathrm{S}, i}\right)}
\end{array}\right.
$$

where, $n$ is the total amount of data, $X_{S, i}$ is the measured snow depth, and $X_{G, i}$ is the snow depth retrieved by GNSS-IR; $\bar{X}_{\mathrm{S}, i}$ is the mean value of the measured snow depth.

\section{Correction of Slope}

When the basic principle of GNSS-IR snow depth inversion was introduced in the previous section, it was assumed that the ground around the station is in an ideal horizontal state. Still, in fact, not all station environments are in a horizontal state. Taking station P351 as an example, there are some obvious upward or downward slopes around the station. Figure 5 shows the digital elevation map around station P351. It can be seen from the figure that there are some different inclinations around station P351. The northeast is relatively gentle with a slope of about $3^{\circ}$, and a downward slope of about $5^{\circ}$ in the northwest. There is an upward slope of about $5^{\circ}$ in the southwest, and an overall downward slope in the southeast direction. Within 0-50 meters, the slope is about $5^{\circ}$, and within 50-100 meters, the slope increases to about $17^{\circ}$. We use the observation data of $\mathrm{P} 351$ station in the range of azimuth $105^{\circ}-150^{\circ}$ and elevation angle $5^{\circ}-30^{\circ}$ to carry out GNSS-IR snow depth inversion. The signal reflection point trajectory in this range is located in the southeast of s station P351 (the lower right corner of the figure), and the farthest radius of the reflection range is about 22 meters. From Figure 5, it can be found that there is a downward slope with a slope of about $5^{\circ}$ in this range, which will have a non-negligible impact on the GNSS-IR snow depth inversion.

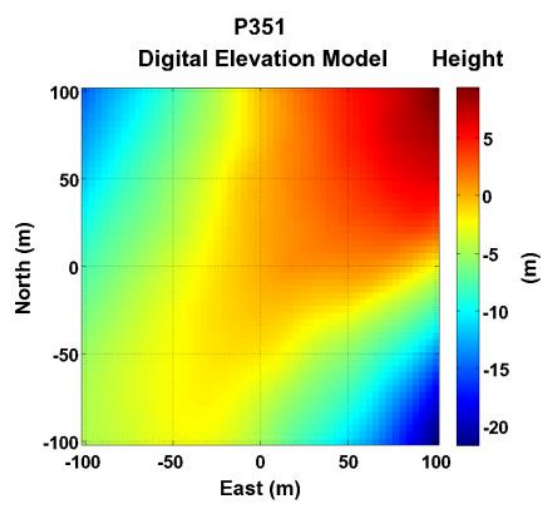

Figure 5. P351 station environment DEM (https://cires1.colorado.edu/portal/?station=P351). 
Figure 6 shows the GNSS-IR snow depth inversion diagram when the ground is sloped downward. $a$ is the slope of the terrain. $E$ is the incident angle of the direct signal, that is the elevation angle of the satellite. $b$ is the angle between the reflected signal and the ground, $b=E+a . h$ is the plumb distance from the antenna phase center to the reflecting surface. $h^{\prime}$ is the vertical distance between the inverted antenna phase and the inclined reflecting surface. There is an angle between $h$ and $h^{\prime}$, and the angle is equal to the slope $a, h=h^{\prime *} \cos a^{12}$.

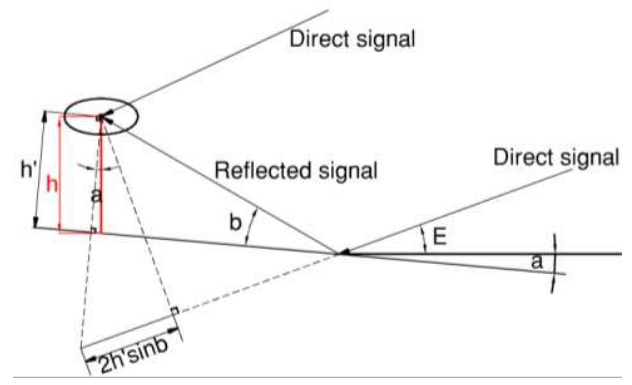

Figure 6. Schematic diagram of GNSS-IR snow depth inversion for sloped.

In order to analyze the relationship between the inversion error caused by slope and slope and satellite elevation angle, this section simulates the inversion error with different slope and elevation angle. The simulation parameters are set as shown in Table 1. It is assumed that the vertical distance from antenna phase center to snow surface is $2 \mathrm{~m}$, the slope is $-5^{\circ}$ (downhill) to $5^{\circ}$ (uphill), and the satellite elevation angle is $5^{\circ}-30^{\circ}$.

Table 1. Simulation parameters of the slope error experiment of the reflecting surface.

\begin{tabular}{|c|c|}
\hline Item & Setting \\
\hline Antenna height & $2 \mathrm{~m}$ \\
\hline Surface materials & Snow \\
\hline Slope & $-5^{\circ} \sim 5^{\circ}$ \\
\hline Elevation angle & $5^{\circ} \sim 30^{\circ}$ \\
\hline
\end{tabular}

Figure 7 shows the simulation results of the inversion error caused by the slope at different slopes and satellite elevation angles. It can be seen from the figure that the inversion error caused by slope is directly proportional to the satellite elevation angle and slope. When the slope is negative, the inversion error is positive, and when the slope is positive, the inversion error is negative. When the slope is constant, the absolute value of the inversion error increases with the elevation angle. When the elevation angle is $30^{\circ}$ and the slope is $5^{\circ}$, the inversion error is about $0.1 \mathrm{~m}$. 


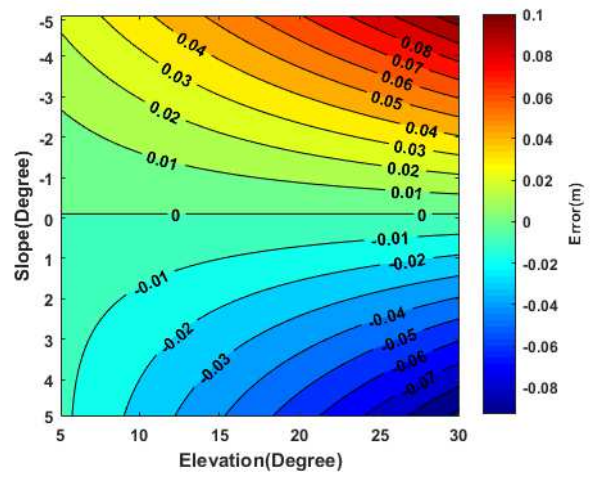

2 Figure 7. Variation of inversion error caused by slope with slope and elevation angle.

Figure 8 shows the residual sequence diagram of the SNR data of the PRN01 satellite before and after adding slope on DOY 225, 2014. In the figure, the blue curve is the SNR residual sequence without slope correction, and the red curve is the SNR residual sequence after slope correction. It can be seen from the figure that the SNR residual sequence move to the right of the x coordinate axis after the slope is added, and its oscillation frequency is higher than when the slope is not added. In the same way, if there is an upward inclination angle on the surface, the SNR residual sequence will move to the left of the $\mathrm{x}$ coordinate axis after adding the surface inclination angle. Its oscillation frequency will be lower than when the inclination slope is not added. It can be found that when there is a downward slope on the surface the satellite altitude angle is less than the reflection angle of the reflected signal. The SNR oscillation frequency drawn from the sinusoidal value of the satellite altitude angle is less than the oscillation frequency drawn by the actual reflection angle. So the retrieved snow depth greater than the measured snow depth. When there is an upward slope on the surface, the satellite elevation angle is greater than the reflection of the reflected signal. At this time, the oscillation frequency of SNR drawn from the sinusoidal value of the satellite elevation angle is greater than that drawn from the actual reflection angle, resulting in the inversion snow depth less than the measured snow depth.
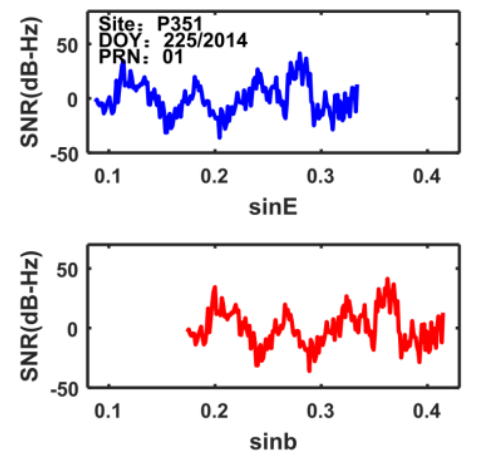

Figure 8. In the top panel, the blue curve is the SNR residual sequence without slope correction, and in the bottom panel, the red curve is the SNR residual sequence after slope correction.

Figure 9 shows the comparison between retrieved snow depth and measured snow depth before and after slope correction from DOY 201, 2014 to DOY 200, 2015. The whole period is divided into the snowfree period (DOY 201-293, 2014, DOY 144-200, 2015), snow growth period (DOY 294-355, 2014), stable snow period (measured snow depth greater than 1.1m) (DOY 356, 2014-DOY 108, 2015) and snow melting period (DOY 109-143, 2015). It can be seen from the figure that the inverted snow depth after slope 
correction is closer to the measured snow depth except for the stable snow period. In the snow-free period, the inverted snow depth is greater than 0, the RMSE before and after the slope correction is $13.6 \mathrm{~cm}$ and $9.4 \mathrm{~cm}$, the RMSE after slope correction is reduced by $30.9 \%$. During the snow growth period and the snow melting period, the inversion snow depth has the best agreement with the actual snow depth, and the snow depth after slope correction has higher accuracy, and the RMSE of the inversion error after slope correction is reduced by $13.1 \%$. During the stable snow period, the inverted snow depth before and after the slope correction is very close, and both are significantly smaller than the measured snow depth. There is a negative error of about $10 \mathrm{~cm}$. The inversion error after the slope correction is slightly larger than that before the correction.

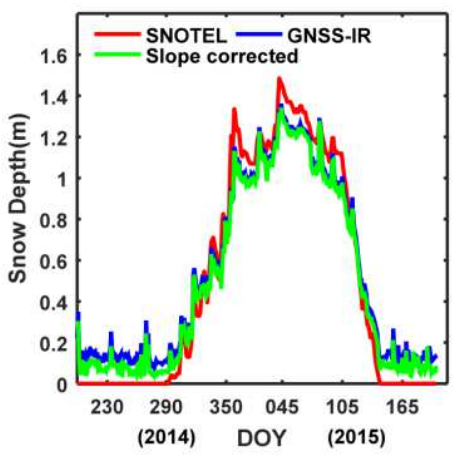

Figure 9. Comparison of inverted snow depth and measured snow depth before and after slope correction sequence after slope correction.

Figure 10 shows the inversion error sequence diagram before and after slope correction during a snowfall period from 2014 to 2015 . In general, the inversion error is basically within $\pm 0.2 \mathrm{~m}$, and the inversion error after correction is closer to zero. In addition, the inversion error is not a random error, but has obvious trend and regularity. In the snow-free period, the error is greater than zero, and the average error before and after slope correction is $13.1 \mathrm{~cm}$ and $8.4 \mathrm{~cm}$, respectively. Then, the error decreases continuously in the growth period of snow until it becomes a negative error, and it shows negative error about $-10 \mathrm{~cm}$ in the stable snow period. After that, error gradually becomes a positive error from snow melting period to the snow free period.

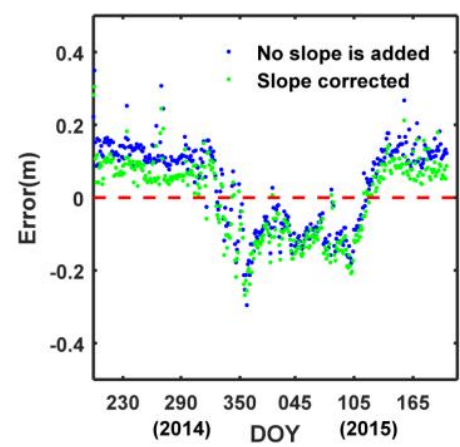

22 Figure 10. Sequence diagram of inversion error before and after slope correction.

Table 2 shows the accuracy comparison of snow depth inversion before and after slope correction in different periods. It can be seen from the table that during the whole snowfall period from 2014 to 2015, 
the accuracy of inversion snow depth after slope correction has been significantly improved, and RMSE decreased from $12.1 \mathrm{~cm}$ to $10.7 \mathrm{~cm}$, which decreased by $11.6 \%$. During the snow-free period, the snow growth period and the snow-melting period, the inversion accuracy after slope correction has been significantly improved, and the accuracy is only slightly reduced during the stable snow period. In general, the accuracy of inversion has been significantly improved after the addition of slope correction, which proves the effectiveness of the slope correction method proposed by us.

7 Table 2. Accuracy statistics of snow depth inversion before and after slope correction in different periods.

\begin{tabular}{|c|c|c|c|}
\hline Period & Method & $\operatorname{RMSE}(\mathrm{m})$ & Decline rate \\
\hline \multirow{2}{*}{$\begin{array}{c}\text { Snow free } \\
\text { period }\end{array}$} & No slope correction & 0.136 & \multirow{2}{*}{$30.9 \%$} \\
\hline & Slope corrected & 0.094 & \\
\hline \multirow{2}{*}{$\begin{array}{c}\text { Snow growth } \\
\text { period }\end{array}$} & No slope correction & 0.101 & \multirow{2}{*}{$7.9 \%$} \\
\hline & Slope corrected & 0.093 & \\
\hline \multirow{2}{*}{$\begin{array}{c}\text { stable snow } \\
\text { period }\end{array}$} & No slope correction & 0.116 & \multirow{2}{*}{$-16.4 \%$} \\
\hline & Slope corrected & 0.135 & \\
\hline \multirow{2}{*}{$\begin{array}{l}\text { Snow melting } \\
\text { period }\end{array}$} & No slope correction & 0.094 & \multirow{2}{*}{$22.3 \%$} \\
\hline & Slope corrected & 0.073 & \\
\hline \multirow{2}{*}{$\begin{array}{l}\text { Whole } \\
\text { inversion } \\
\text { period }\end{array}$} & No slope correction & 0.121 & \multirow[b]{2}{*}{$11.6 \%$} \\
\hline & Slope corrected & 0.107 & \\
\hline
\end{tabular}

\section{8}

\section{Prediction and Correction of Inversion Error Based on BPNN}

Combining the snow depth comparison chart in Fig. 9 with the error analysis chart in Fig. 10, we find that the inverted snow depth is greater than the measured snow depth when the measured snow depth is zero. As the snow depth increases, the snow depth of the inversion error also decreases to zero, and then becomes a negative error. The absolute value of the error continues to increase, indicating that there is a certain correlation between the inversion error and the snow depth.

In order to further explore the relationship between the inverted snow depth and the measured snow depth, Figure 11 shows a comparison diagram of the inverted snow depth value and error during the three snowfall periods from DOY 201, 2011 to DOY 200, 2014. It can be seen from the figure that the error also increases gradually with the increase of snow depth, showing a significant correlation between the two. In the three snowfall periods, when the retrieved snow depth values are the same, the corresponding error values are basically the same. Therefore, according to the known relationship between the retrieved snow depth and the error, we can predict and correct the error in the next snow period inversion. Therefore, the accuracy of GNSS-IR snow depth inversion could be improved.

We introduce the BPNN to study and analyze the relationship between the inversion of snow depth and the error. Based on the correction of the slope, it uses 1006 sets of three consecutive snow periods from DOY 201, 2011 to DOY 200, 2014. The data constructs a BPNN and conducts training. The trained neural network is used to predict the errors of 360 retrieved snow depth values from DOY 201, 2014 to DOY 200, 2015, and the corrected snow depth inversion value can be obtained by subtracting the predicted error value from the retrieved snow depth. Through a large number of experiments, we try to determine the best hidden layer number of neural network. 


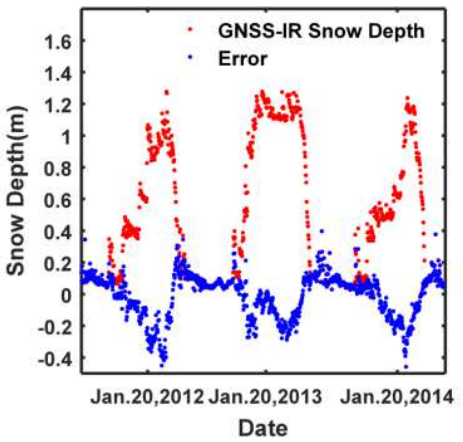

1

Figure 11. Comparison of inverted snow depth values and errors from 2011 to 2014.

Figure 12 shows the comparison between the original error and prediction error of snow depth inversion in the snow period from 2014 to 2015. It can be seen from the figure that the predicted snow depth inversion error after BPNN training is very close to the real snow depth inversion error, and the changing trend of prediction error and real error is completely consistent, their correlation is 0.894 . Therefore, after correcting the snow depth with the most inversion of the prediction error, the inversion error can be greatly eliminated, and the accuracy of snow depth inversion can be improved.

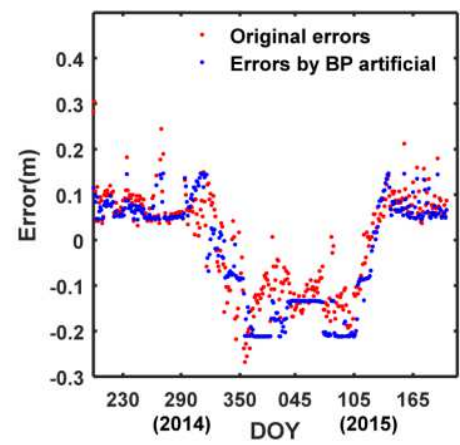

Figure 12. Comparison of inversion error and BPNN prediction error in snowfall period from 2014 to 2015.

Figure 13 shows the comparison of measured snow depth, GNSS-IR retrieved snow depth and GNSS-IR retrieved snow depth corrected by BPNN 2014-2015 snowfall period. It can be seen from the figure that the agreement between the GNSS-IR inverted snow depth and the measured snow depth after the BPNN prediction error correction is significantly improved compared to the inverted snow depth without error correction. In the snow-free period, the effect of the error correction is obvious. The snow depth inversion is very close to zero, and the RMSE is $2.9 \mathrm{~cm}$, which is $69.1 \%$ lower than that without the correction, showing a high accuracy. The RMSE decreases by $14 \%$ and $32.9 \%$ in the snow growth period and snow melting period. In the stable snow period, the retrieved snow depth after correction also has good accuracy; the RMSE decreases from $0.135 \mathrm{~cm}$ to $0.069 \mathrm{~cm}$ with a decrease of $48.9 \%$. 


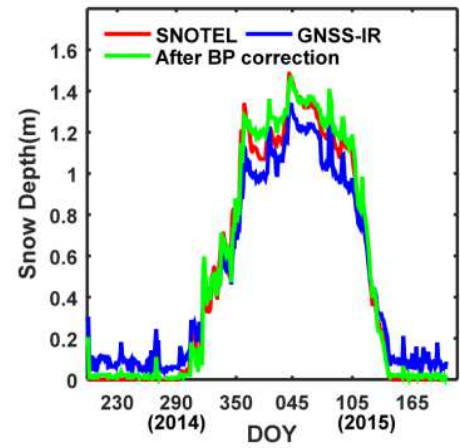

2 Figure 13. Comparison of BPNN prediction error before and after correction.

Table 3 shows the accuracy comparison after a single slope correction and the accuracy comparison results before and after the double correction of the slope + BPNN prediction error. It can be seen from the table that the accuracy of snow depth inversion is obviously better than that of single slope correction through simultaneous correction of slope and BPNN prediction error in each stage of the whole snowfall period. Among them, the accuracy of stable snow period is the most obvious. The accuracy of single slope correction is $16.4 \%$ lower than that of uncorrected, the accuracy of slope + BPNN correction is $48.9 \%$ higher than that of uncorrected and $49.3 \%$ higher than the accuracy of single slope correction. During the snow growth period, the accuracy improvement rate increased from $7.9 \%$ to $14.9 \%$, which was relatively small. Compared with single correction, the accuracy of the double correction is significantly improved in snow-free periods and snow melting periods. In the whole snow period, the RMSE decreased from 0.121 $\mathrm{m}$ to $0.107 \mathrm{~m}$ after the single slope correction, and the accuracy increased by $11.6 \%$. After the double correction of slope and BPNN prediction error, the RMSE decreased from $0.121 \mathrm{~m}$ to $0.057 \mathrm{~m}$, and the accuracy increased by $52.9 \%$. The results show that the two error improvement methods can significantly improve the accuracy of GNSS-IR snow depth inversion.

17 Table 3. Comparison of prediction error correction accuracy of BPNN.

\begin{tabular}{|c|c|c|c|}
\hline Period & Method & RMSE(m) & Decline rate \\
\hline \multirow{3}{*}{ Snow free period } & No correction & 0.136 & $\backslash$ \\
\cline { 2 - 4 } & Slope & 0.094 & $30.9 \%$ \\
\cline { 2 - 4 } & Slope + BPNN & 0.029 & $78.7 \%$ \\
\hline \multirow{3}{*}{ Snow growth period } & No correction & 0.101 & $\backslash$ \\
\cline { 2 - 4 } & Slope & 0.093 & $7.9 \%$ \\
\cline { 2 - 4 } & Slope + BPNN & 0.086 & $14.9 \%$ \\
\hline \multirow{3}{*}{ Snow stable period } & No correction & 0.116 & $\backslash$ \\
\cline { 2 - 4 } & Slope & 0.135 & $-16.4 \%$ \\
\cline { 2 - 4 } Snow melting period & Slope + BPNN & 0.069 & $48.9 \%$ \\
\hline & No correction & 0.094 & $\backslash$ \\
\cline { 2 - 4 } & Slope & 0.073 & $22.3 \%$ \\
\cline { 2 - 4 } & Slope + BPNN & 0.049 & $47.9 \%$ \\
\hline \multirow{3}{*}{ Whole inversion period } & No correction & 0.121 & 1 \\
\cline { 2 - 4 } & Slope & 0.107 & $11.6 \%$ \\
\cline { 2 - 4 } & Slope + BPNN & 0.057 & $52.9 \%$ \\
\hline
\end{tabular}


The above two sets of experiments were carried out from two aspects: the influence of the slope on GNSSIR snow depth inversion and the relationship between snow depth inversion and inversion error. The experimental results show that the retrieval error of GNSS-IR snow depth is directly proportional to the slope angle and elevation angle. When the antenna phase center is $2 \mathrm{~m}$ away from the reflector, the elevation angle is $30^{\circ}$, and the slope angle is $5^{\circ}$, the GNSS-IR snow depth inversion error is about $0.1 \mathrm{~m}$, so the error caused by the slope to GNSS-IR snow depth inversion cannot be ignored. Through the long time series GNSS-IR snow depth inversion results, we can find that with the increase of snow depth, the distance between snow surface and antenna phase center is getting closer and closer. This will make the separation of direct signal and reflected signal more difficult. Therefore, in order to ensure the accuracy of GNSS-IR snow depth inversion, the height of the GNSS tracking station should be increased as much as possible. It can not only improve the accuracy of snow depth inversion but also increase the reflection area and improve the coverage of monitoring. However, it also means that the surface condition in the covered reflector may be more complex. When the antenna height is higher, the same ground slope angle will cause more significant error. Therefore, slope correction is one of the essential measures to ensure the accuracy of GNSS-IR snow depth inversion.

Through the GNSS-IR snow depth inversion experiment of long time series, it can be found that there is a significant correlation between the snow depth and the error. With the increase of snow depth, the snow depth inversion error also increases. Moreover, in different snowfall periods, the correlation between snow depth and inversion error is basically the same. Therefore, BPNN is used to train the existing inversion snow depth and inversion error, and establish the model between them to predict and correct the error in the next snow period. In the experiment above, the accuracy of snow depth inversion is improved significantly after the prediction error and correction of the BPNN. However, there are still some errors between the retrieved snow depth and the measured snow depth, which shows that there are still some limitations in the use of the BPNN in error prediction. The reason is not the limitations of the BPNN, but the error source that we could not find. Through GNSS-IR snow depth inversion of long time series, it can be found that the error increases with the increase of snow depth, which is a real phenomenon, and the relationship between the retrieved snow depth and the error value is basically consistent in different snowfall periods. Therefore, we can predict and correct the error of snow depth inversion in the next period through the existing inversion snow depth and error. However, it is only corrected according to the law of error. Although the accuracy is improved significantly, it is not analyzed and corrected from the source of error. Given this phenomenon, we consider that there are two primary sources of error after summarizing the existing research results. On the one hand, the distance between the snow surface and the antenna phase center gradually decreases with the increase of snow depth, which makes the separation accuracy of direct signal SNR and reflection SNR decrease, resulting in snow depth inversion error. In this respect, the error may be reduced by looking for more effective signal separation technology or increasing the height of the station. On the other hand, the signal passes through the snow surface and then reflects into the receiver to produce a negative error with the increase of snow depth. This has been verified by the existing experimental results. With the increase of snow depth, the main surface error of snow depth inversion is negative. However, the ability of the signal to penetrate the snow and reflect again after penetration needs further quantitative research. Therefore, it will be the focus of future research to analyze the deep reason for the error increasing with the increase of snow depth and correct the error source, which is also an effective means to improve the accuracy of snow depth inversion further.

\section{Conclusions}

As an indispensable freshwater resource in the earth' $\mathrm{s}$ ecological environment, the change of snow reserves is not only affected by the earth' $\mathrm{s}$ ecological environment, but also reacts on the earth' $\mathrm{s}$ ecological environment. Taking P351 station in the United States as an example, we analyze the causes of 
errors in GNSS-IR snow depth inversion caused by slope and puts forward the correction method. Based on the analysis of the rules of snow depth and snow depth inversion error, a BPNN method is proposed to forecast and correct the snow depth inversion error in the next snowfall period. The accuracy of the inversion results is significantly improved, and the following conclusions are obtained:

(1) We analyze the relationship between inversion error and slope and satellite elevation angle. The results show that the retrieval error of snow depth caused by slope is directly proportional to the absolute value of slope and elevation angle. When the antenna phase center is $2 \mathrm{~m}$, the absolute value of the slope is $5^{\circ}$, and the elevation angle is $30^{\circ}$, the inversion error can reach $0.1 \mathrm{~m}$. The error caused by the slope is an essential source of GNSS-IR snow depth inversion error. Then, taking the surrounding environment of P351 station as an example, we carried out a GNSS-IR snow depth inversion experiment of slope correction, and the results showed that the corrected snow depth inversion accuracy increased by $11.6 \%$.

(2) We use BPNN to train the snow depth inversion value and inversion error in the three snowfall period from 2011 to 2014. It predicts and corrects the snow depth inversion error during the snowfall period from 2014 to 2015. The results show that after the error correction, the RMSE of the snow depth inversion decreased from $0.107 \mathrm{~m}$ to $0.057 \mathrm{~m}$, and the correlation coefficient increased from 0.994 to 0.996 .

(3) After simultaneously correcting the slope and BPNN prediction error, the RMSE of the snow depth inversion decreased from $0.121 \mathrm{~m}$ to $0.057 \mathrm{~m}$, the correlation coefficient was increased from 0.994 to 0.996 , and the accuracy increased by $52.9 \%$. This shows that the slope correction and BPNN prediction error correction can significantly improve the accuracy of GNSS-IR snow depth inversion.

(4) The slope of P351 station is relatively simple, it is only one downward slope in the selected azimuth of $105^{\circ}-150^{\circ}$. How to correct the more complex slope will be one of the focuses of research work. At the same time, there is an apparent correlation between retrieved snow depth and inversion error. The analysis and research on the causes of this relationship are also the critical work to improve the accuracy of GNSSIR snow depth inversion.

\section{References}

1. M. Martin-Neira. A passive reflectometry and interferometry system (PARIS): application to ocean altimetry. ESA journal, vol. 17, no. 4, pp. 331-355(1993).

2. A. Bilich, and K. M. Larson. Mapping the GPS multipath environment using the signal-to-noise ratio (SNR). Radio Science, vol. 42:RS6003,(2007).

3. A. Bilich, K. M. Larson and P. Axelrad. Modeling GPS phase multipath with SNR: case study from the salar de uyuni, boliva. Journal of Geophysical Research, vol.113, no. B4, pp. B04401(2008).

4. C. J. Comp and P. Axelrad. Adaptive SNR-based carrier phase multipath mitigation technique. IEEE Transactions on Aerospace and Electronic Systems, vol. 34, no. 1, pp. 264-276(1998).

5. A. El-Fallahi, Rafael Martí and L. Lasdon. Path relinking and GRG for artificial neural networks. European Journal of Operational Research, vol. 169, no. 2, pp. 508-519(2006).

6. A. Hu and K. Zhang. Using bidirectional long Short-Term memory method for the height of F2 peak forecasting from Ionosonde Measurements in the Australian region. Remote Sensing, vol. 10, no. 10, pp. 1658(2018).

7. K. M. Larson, E. D. Gutmann. V. U. Zavorotny, J. J. Braun and F. G. Nievinski. Can we measure snow depth with GPS receivers?. Geophysical Research Letters, vol. 36, no. 17, pp. L17502(2009). 
8. K. M. Larson, J. S. Loefgren, and R. Haas. Coastal sea level measurements using a single geodetic GPS receiver. Advances in Space Research, vol. 51, no. 8, pp. 1301-1310(2013).

9. J. S. Löfgren, R. Haas, and H. G. Scherneck. Sea level time series and ocean tide analysis from multipath signals at five GPS sites in different parts of the world," Journal of Geodynamics, vol. 80, pp. 66-80, Oct. 2014.

10. K. M. Larson, J. Wahr and P. Kuipers Munneke. Constraints on snow accumulation and firn density in Greenland using GPS receivers. Journal of Glaciology, vol. 61, no. 225, pp. 101-114(2014).

11. Y. Li, X. Chang, K. Yu, S. Wang and J. Li. Estimation of snow depth using pseudorange and carrier phase observations of GNSS single-frequency signal. GPS Solutions, vol. 23, no. 4(2019).

12. Z.Li, P. Chen, N. Zheng and H. Liu. Accuracy analysis of GNSS-IR snow depth inversion algorithms. Advances in Space Research, to be published. DOI:10.1016/j.asr.2020.11.021.

13. F. G. Nievinski and K. M. Larson, "Inverse modeling of GPS multipath for snow depth estimationPart II: Application and validation," IEEE Transactions on Geoscience and Remote Sensing, vol. 52, no. 10, pp. 6564-6573, Oct. 2014.

14. M. Ozeki and K. Heki. GPS snow depth meter with geometry-free linear combinations of carrier phases. Journal of Geodesy, vol. 86, no. 3, pp. 209-219(2011).

15. X. Qian and S. Jin. Estimation of snow depth from glonass snr and phase-based multipath reflectometry. IEEE Journal of Selected Topics in Applied Earth Observations and Remote Sensing, vol. 9, no. 10, pp. 4817-4823(2016).

16. B. K. Rani, K. Srinivas and A. Govardhan. Rainfall prediction with TLBO optimized ANN. Journal of scientific Industrial Research, vol. 73, no. 10, pp. 643-647(2014).

17. C. Roesler, K. M. Larson. Software tools for GNSS interferometric reflectometry (GNSS-IR). GPS Solutions, vol. 22, no. 3, pp. 80(2018).

18. E. E. Small, K. M. Larson and J. J. Braun. Sensing vegetation growth with reflected GPS signals. Geophysical Research Letters, vol. 37, no. 12(2010).

19. W. Wan, K. M. Larson, E. E. Small, C. C. Chew and J. J. Braun. Using geodetic GPS receivers to

20. H. Wei, X. He, Y. Feng, S. Jin and F. Shen. Snow depth estimation on slopes using GPS-Interferometric

21. X. Wang, S. Zhang, L. Wang, X. He and Q. Zhang. Analysis and combination of multi-GNSS snow depth retrievals in multipath reflectometry. GPS Solutions, vol. 24, no. 77(2020).

22. K. Yu, S. Wang, Y. Li, X. Chang and J. Li. Snow depth estimation with GNSS-R dual receiver observation. Remote Sensing, vol. 11, no. 17, pp. 2056(2018).

35 The authors declare no competing interests.

36 Acknowledgement 
1 The authors would like to thank the $\mathrm{PBO} \mathrm{H} 2 \mathrm{O}$ research team for providing experimental data 2 (https://cires1.colorado.edu/portal/), and the US Department of Agriculture (USDA) Natural Resources

3 Conservation Service Organization (NRCS) for providing measured snow depth data 4 (https://www.wcc.nrcs.usda.gov/snow/). This study was funded by the National Natural Science 5 Foundation of China (41404031) and Outstanding Youth Science Fund of Xi' an University of Science 6 and Technology (2018YQ2-10).

\section{Author contributions}

8 P.C. and Z. L. conceived the experiments. Z.L. and N.Z. conducted the experiments, and Z.L. performed 9 statistical analysis and figure generation. P.C. and Z.L. wrote the main manuscript text. All authors 10 reviewed the manuscript. 


\section{Figures}

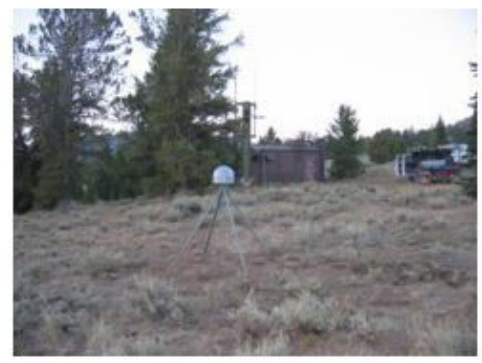

(a)

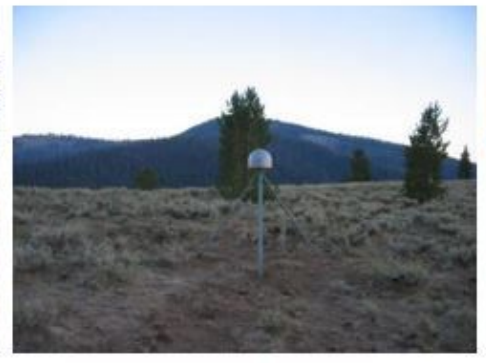

(b)

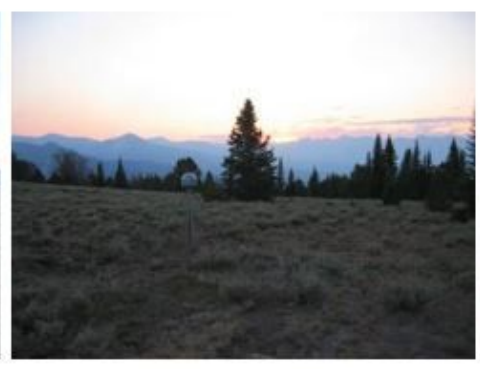

(b)

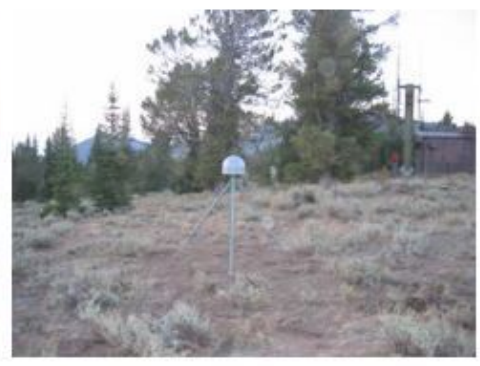

(d)

\section{Figure 1}

Environment map of P351 station. (a), (b), (c) and (d) shows the topography of east, west, north and south view of the station, respectively. (https://www.unavco.org/instrumentation/networks/status/ nota/photos/P351)

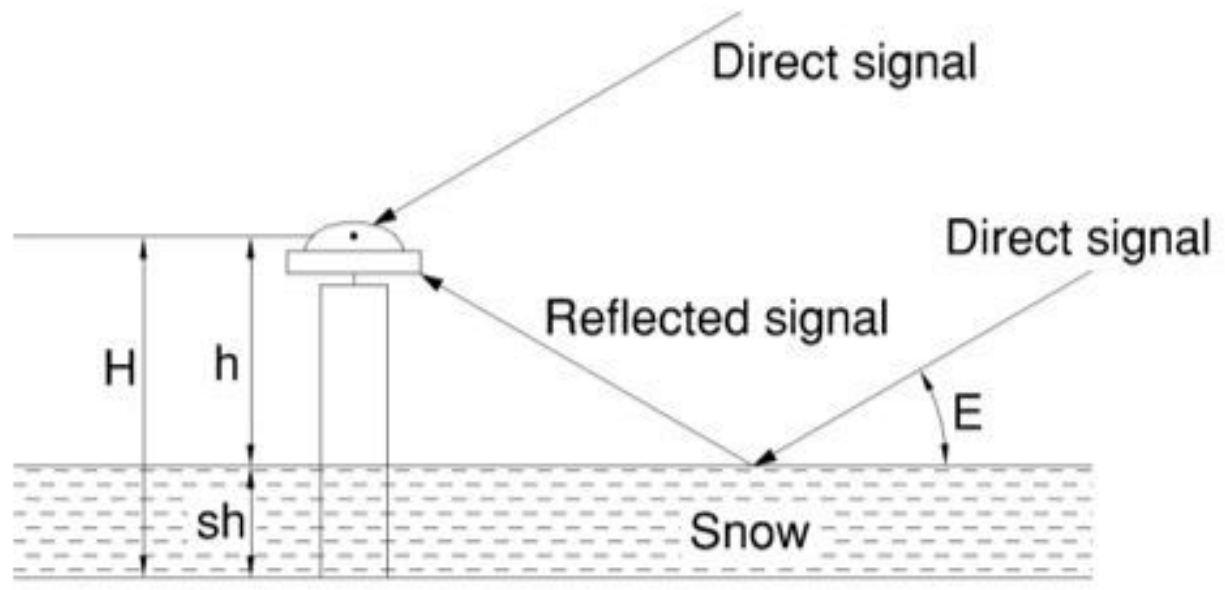

Figure 2

Schematic diagram of GNSS-IR snow depth inversion. 

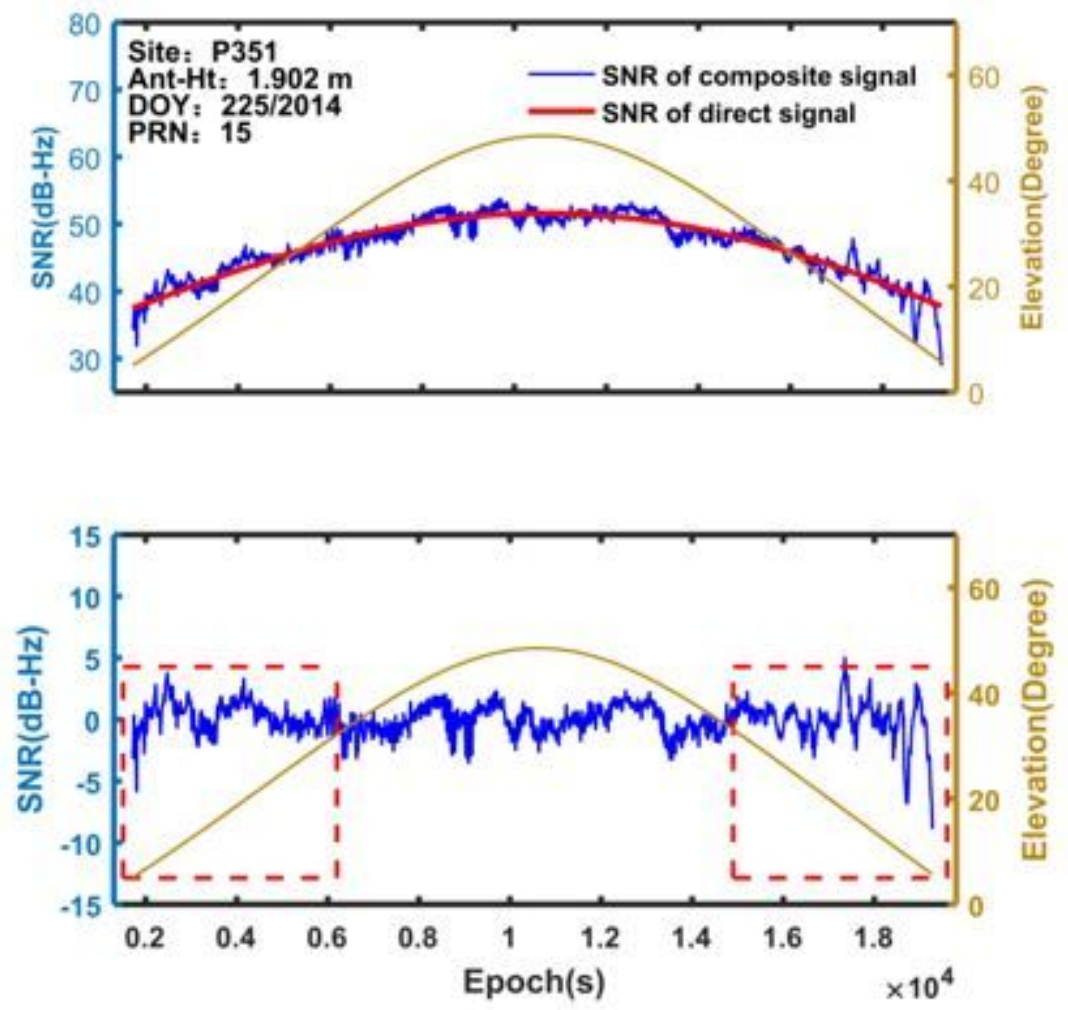

Figure 3

A sequence of signal SNR in a continuous observation arc of PRN15 satellite on DOY 225, 2014. 


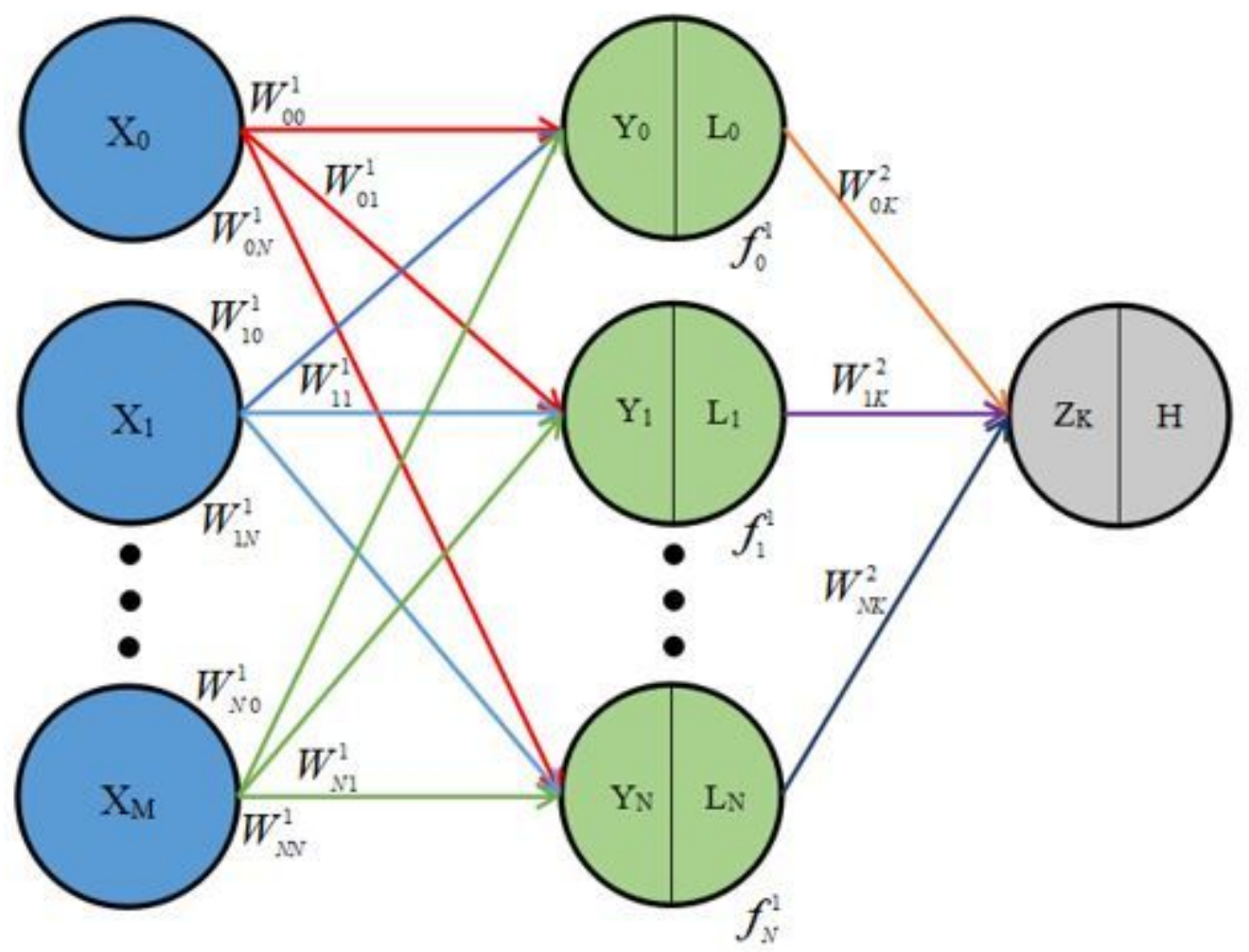

Input layer

Hidden layer

Output layer

Figure 4

Schematic diagram of BPNN topology.

\section{P351}

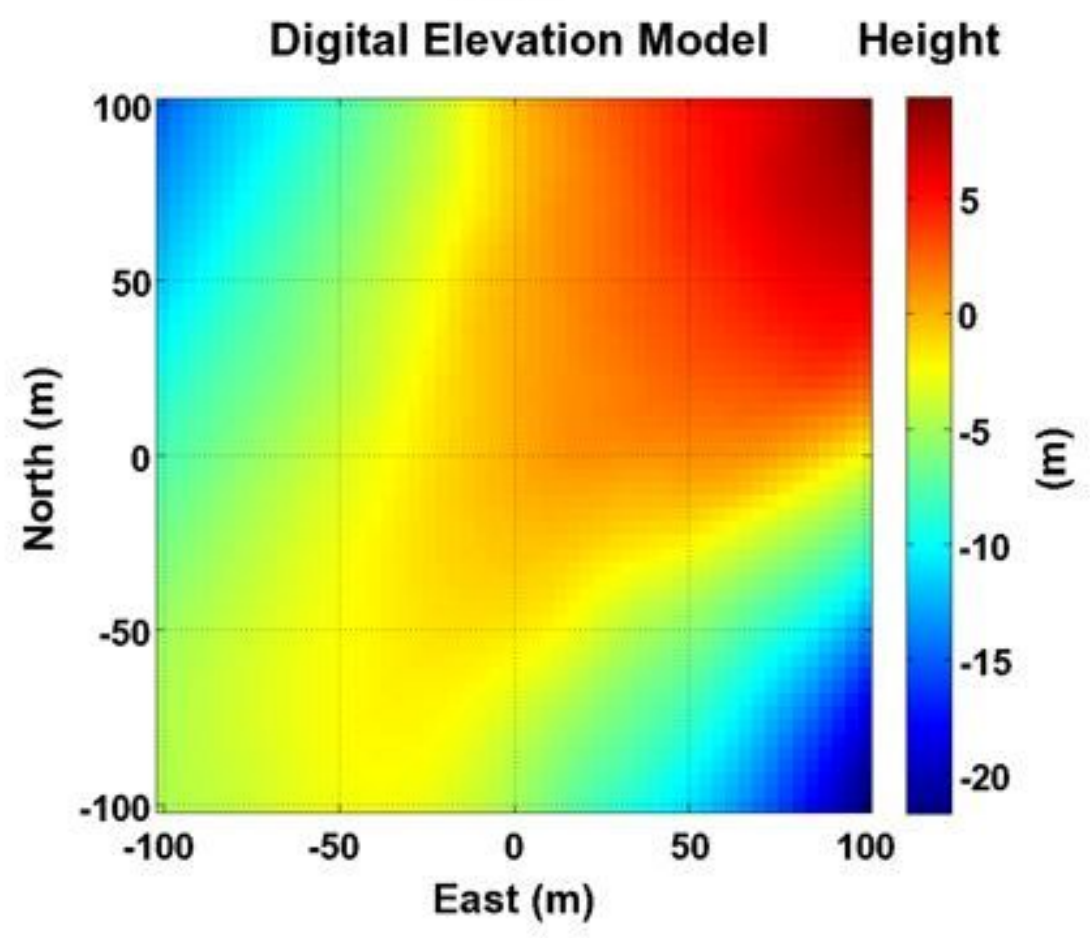


Figure 5

P351 station environment DEM (https://cires1.colorado.edu/portal/?station=P351).

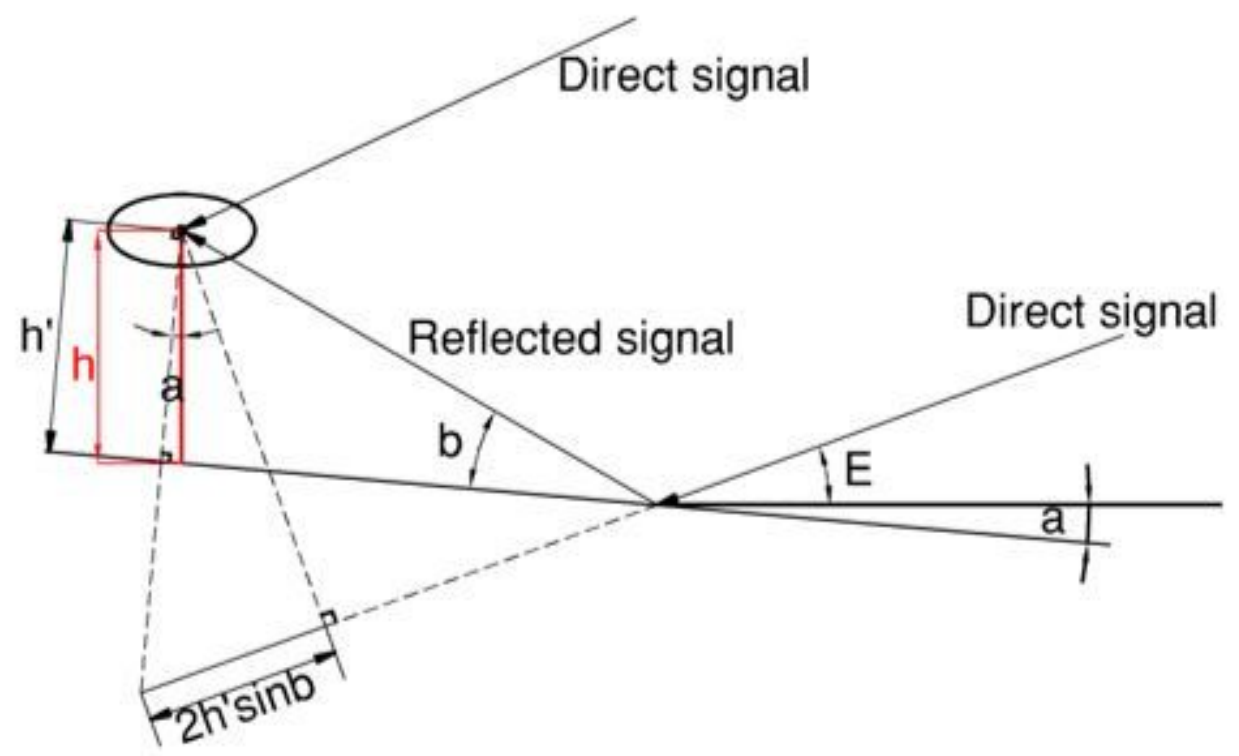

Figure 6

Schematic diagram of GNSS-IR snow depth inversion for sloped.

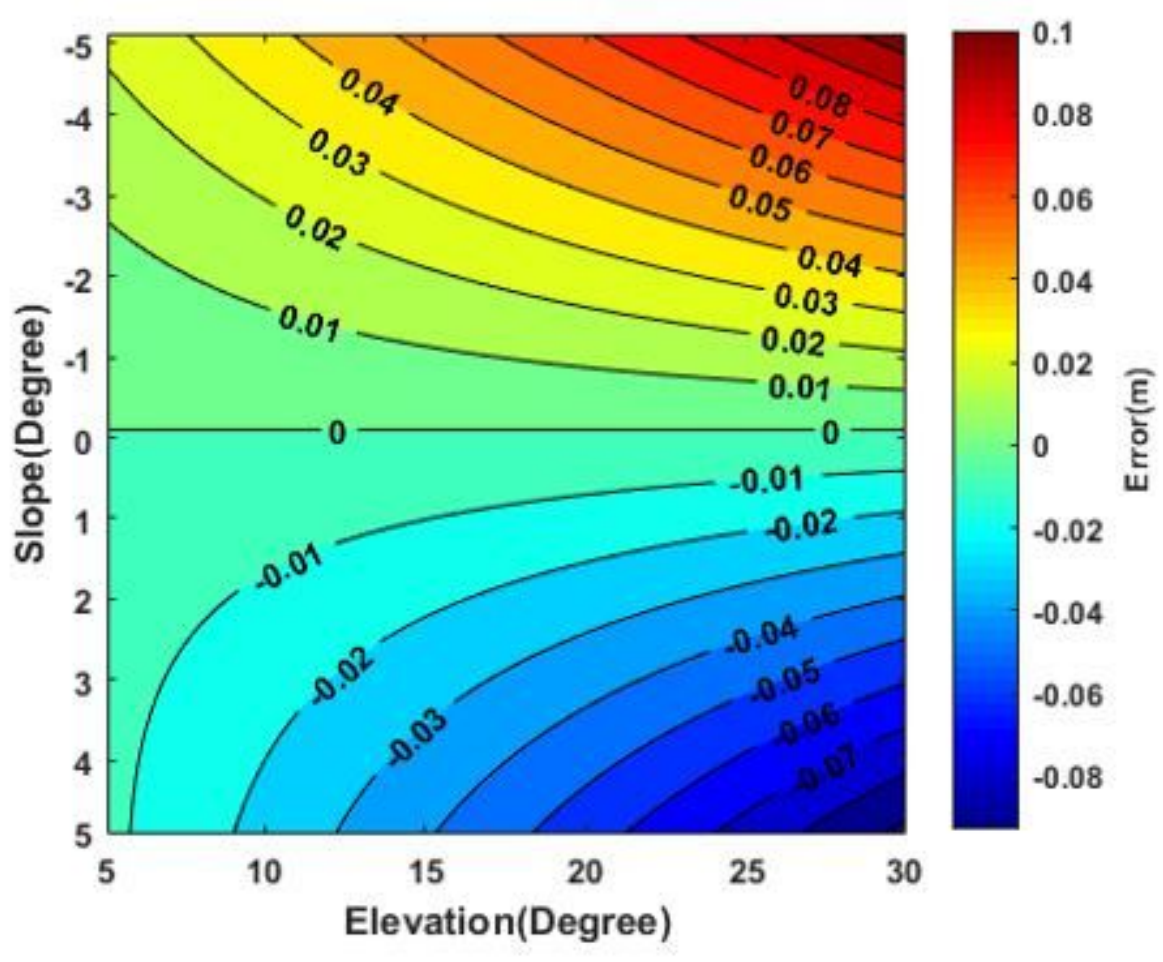

Figure 7 
Variation of inversion error caused by slope with slope and elevation angle.
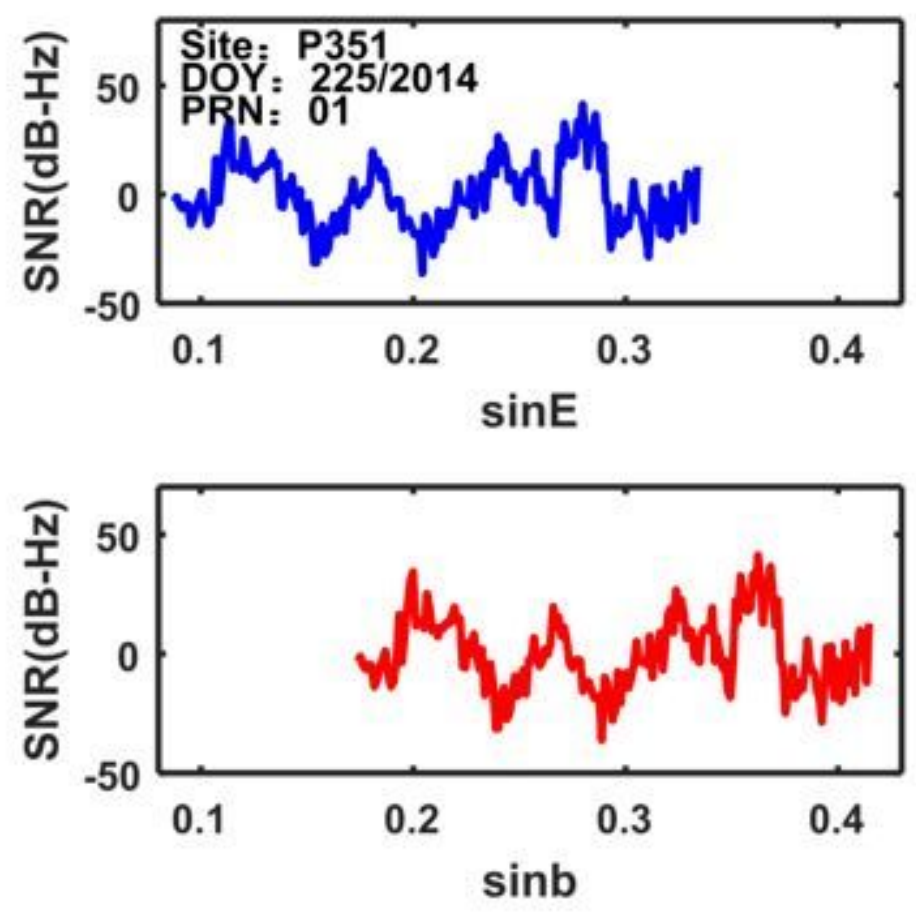

Figure 8

In the top panel, the blue curve is the SNR residual sequence without slope correction, and in the bottom panel, the red curve is the SNR residual sequence after slope correction. 


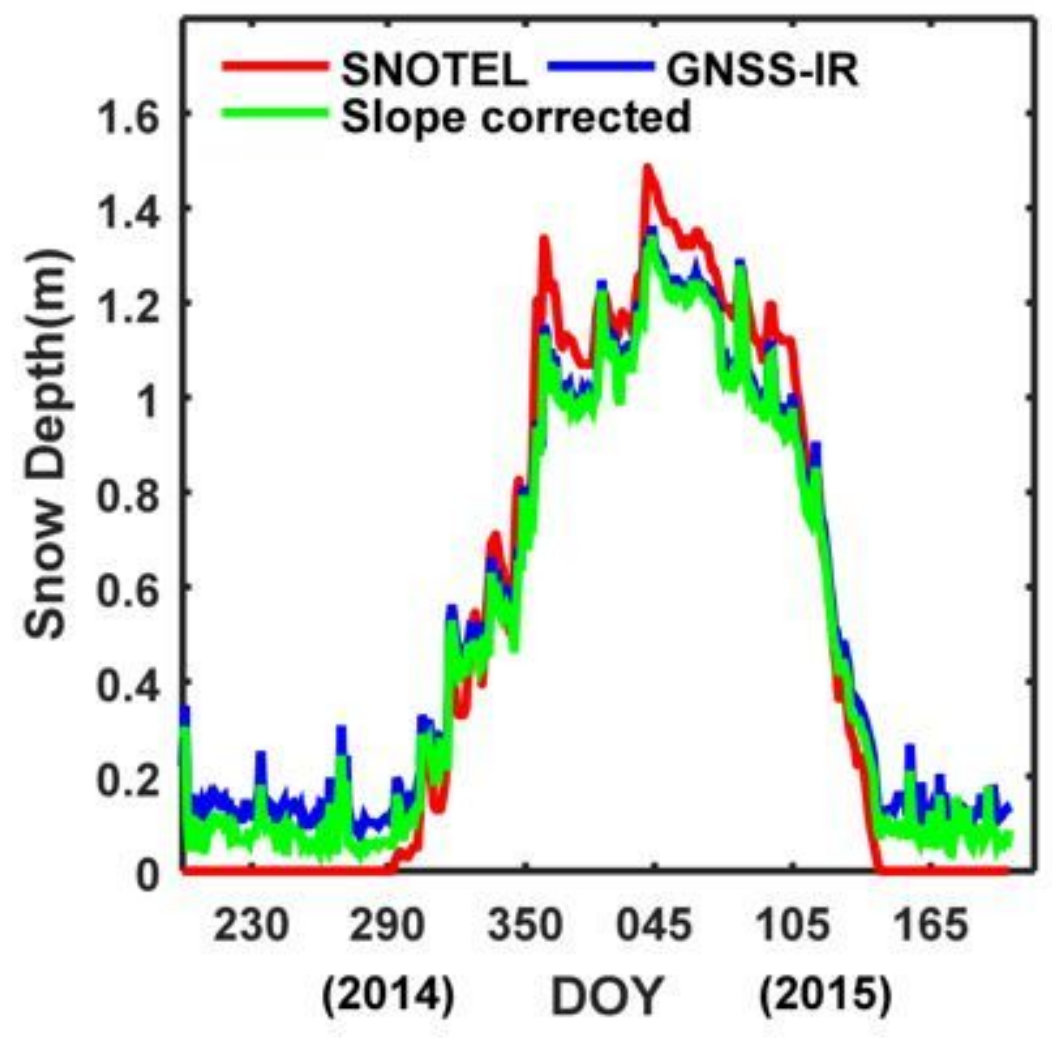

Figure 9

Comparison of inverted snow depth and measured snow depth before and after slope correction sequence after slope correction. 


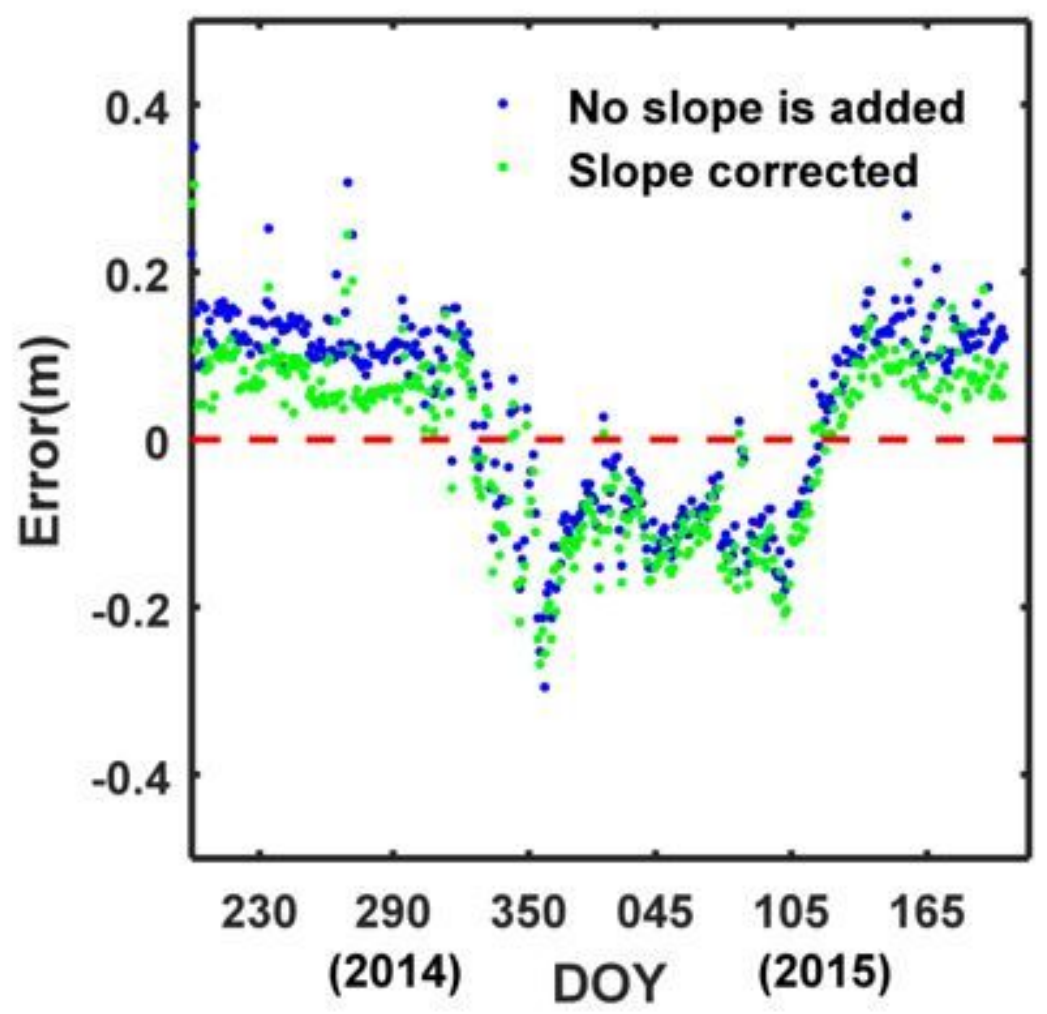

Figure 10

Sequence diagram of inversion error before and after slope correction.

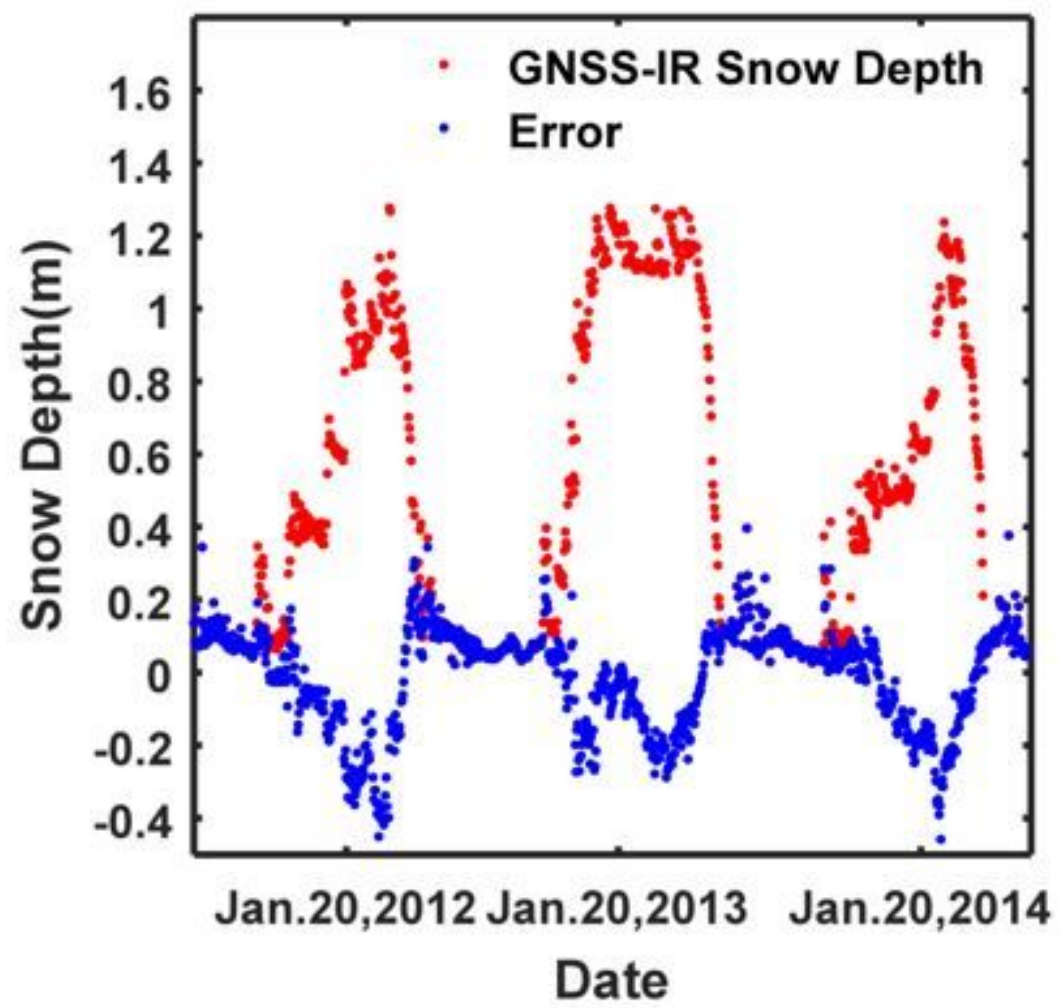


Figure 11

Comparison of inverted snow depth values and errors from 2011 to 2014 .

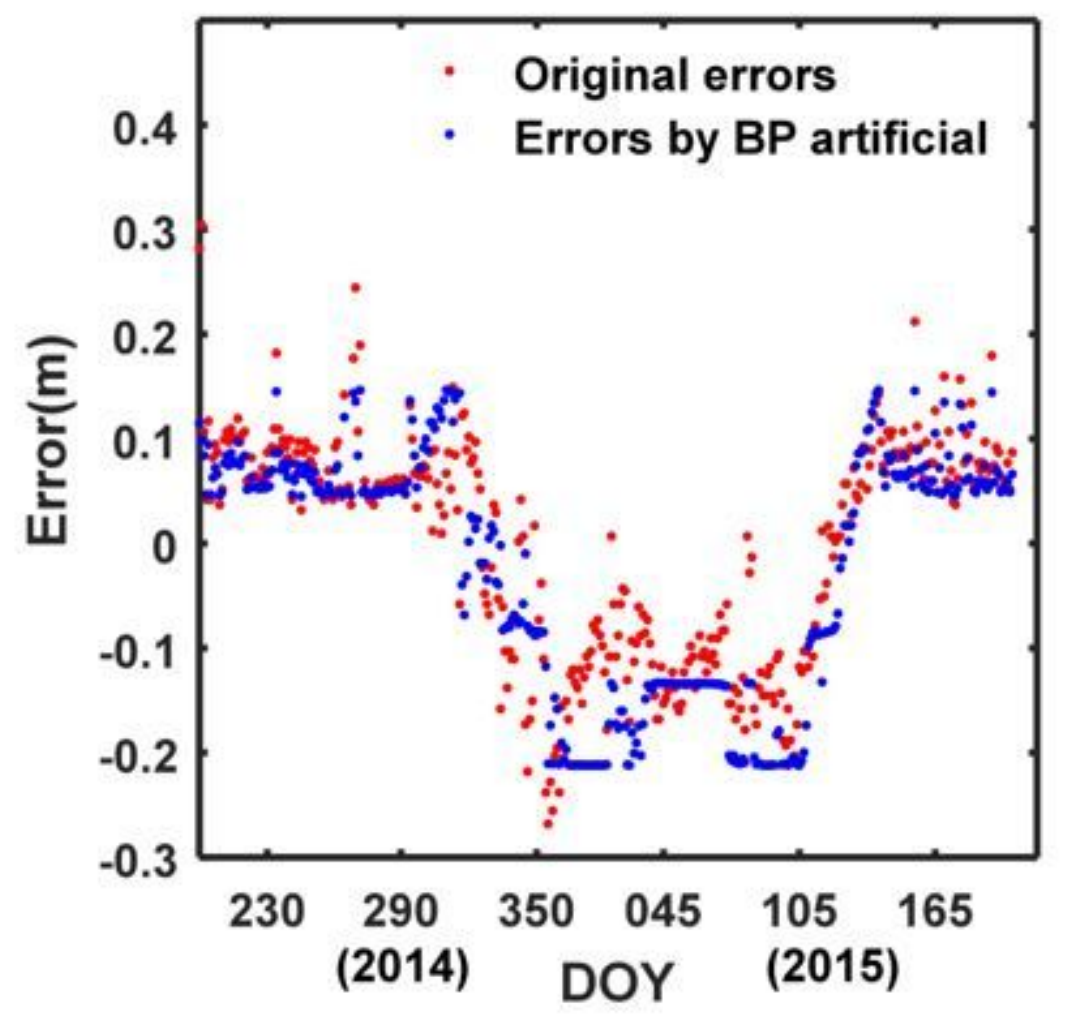

Figure 12

Comparison of inversion error and BPNN prediction error in snowfall period from 2014 to 2015. 


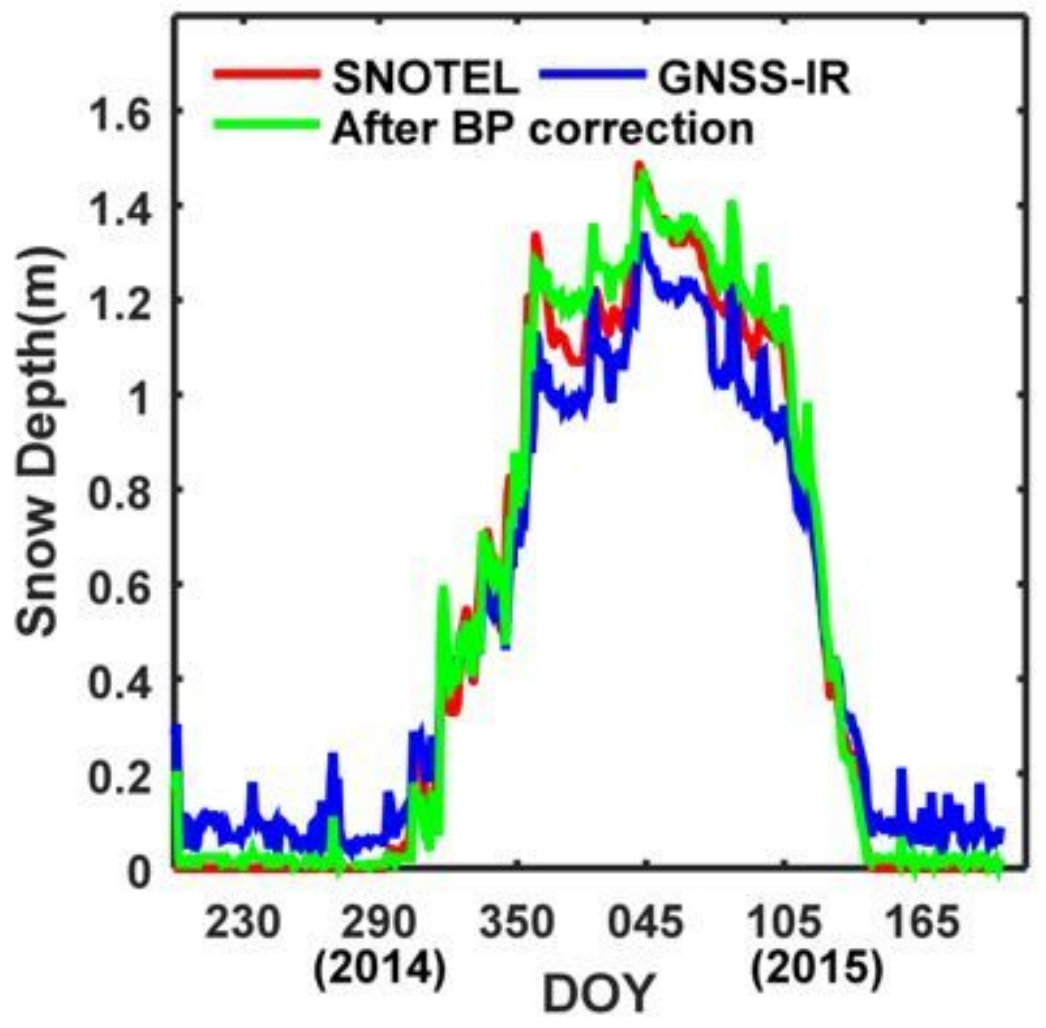

Figure 13

Comparison of BPNN prediction error before and after correction. 